\title{
Detection of rhinovirus in induced sputum at exacerbation of chronic obstructive pulmonary disease
}

\author{
T.A.R. Seemungal*, R. Harper-Owen*, A. Bhowmik*, D.J. Jeffries**, J.A. Wedzicha*
}

Detection of rhinovirus in induced sputum at exacerbation of chronic obstructive pulmonary disease. T.A.R. Seemungal, R. Harper-Owen, A. Bhowmik, D.J. Jeffries, J.A. Wedzicha. (C) ERS Journals Ltd 2000.

ABSTRACT: Common colds are associated with exacerbations of chronic obstructive pulmonary disease (COPD). However, the role of the common cold virus (human rhinovirus) in the production of symptoms and lower airway inflammation at COPD exacerbation is unknown.

Thirty three patients with moderate-to-severe COPD were seen at baseline, when the number of chest infections in the previous year was noted, and acutely at COPD exacerbation. Within $48 \mathrm{~h}$ after the onset of the exacerbation and at baseline, nasal aspirates and induced sputum were taken for rhinovirus reverse transcriptase polymerase chain reaction (RT-PCR) analysis and determination of cytokine levels. Symptoms, recorded on diary cards, were noted and forced expiratory volume in one second (FEV1) and forced vital capacity (FVC) measured.

At exacerbation, mean FEV1 and FVC fell significantly from baseline $(p<0.001)$. Ten of 43 exacerbations were associated with rhinovirus infection, detected in induced sputum. In four of these, nasophageal samples contained no detectable rhinovirus. All baseline samples were negative for rhinovirus. The simultaneous presence of increased nasal discharge/nasal congestion (in 26 of the 43 exacerbations) and increased sputum (29 exacerbations) was strongly associated with the presence of rhinovirus (odds ratio $6.15 ; \mathrm{p}=\mathbf{0 . 0 3 6}$ ). Total symptom scores were greater for rhinovirus as compared to nonrhinovirus exacerbations $(\mathrm{p}=\mathbf{0 . 0 3 9})$. Median baseline sputum interleukin-6 levels rose from 90.2 to $140.3 \mathrm{pg} \cdot \mathrm{mL}^{-1}$ at exacerbation $(p=0.005)$; the change was greater in the presence of rhinovirus infection $(p=0.008)$.

Rhinovirus infection can be detected at chronic obstructive pulmonary disease exacerbation. This is associated with elevation of lower airway interleukin-6 levels, which may mediate lower airway symptom expression during chronic obstructive pulmonary disease exacerbations.

Eur Respir J 2000; 16: 677-683.

\author{
*Academic Respiratory Medicine and \\ **Virology, Dept of Medical Micro- \\ biology, St. Bartholomew's and Royal \\ London School of Medicine and \\ Dentistry, London, UK.
}

Correspondence: J.A. Wedzicha, Academic Respiratory Medicine, Dominion House, St Bartholomew's Hospital, West Smithfield, London EC1A 7BE, UK. Fax: 44 2076018616

Keywords: Chronic obstructive pulmonary disease exacerbation colds

frequent exacerbators

interleukin-6

rhinovirus

Received: December 301999

Accepted after revision May 292000

R. Harper-Owen was funded by the Joint Research Board, St. Bartholomew's Hospital Special Trustees. T. Seemungal was in receipt of a British Lung Foundation Fellowship.
Patients with moderate-to-severe chronic obstructive pulmonary disease (COPD) are prone to exacerbations, associated with acute deterioration in symptoms and lung function, which increase in frequency with increasing severity of COPD $[1,2]$. COPD exacerbations have major effects on health status [3], are associated with considerable morbidity and mortality, and are frequently triggered by upper respiratory tract infections [4]. These exacerbations are associated with increased airway inflammation and airflow obstruction $[4,5]$. Studies in childhood asthma have shown that viruses, especially rhinovirus (the cause of the common cold), can be detected by polymerase chain reaction (PCR) in nasopharyngeal samples from a large proportion of these exacerbations [6]. However, the role of rhinovirus in COPD exacerbation has not been evaluated using PCR methods.

There has been considerable debate as to the mechanisms of virally induced exacerbations in patients with COPD. One possibility is that airway cytokines produced by the upper airway during infection can produce lower airway inflammation [7]. However, a study has reported that rhinovirus ribonucleic acid (RNA) can be detected in the lower airway by PCR after experimental nasal rhino- virus inoculation, suggesting that rhinovirus can directly infect the lower airway [8].

Although there is evidence to suggest that viruses are important in triggering COPD exacerbation [9], there is no information as to whether rhinovirus can be isolated from the lower airway during COPD exacerbation and its relation to airway inflammation at exacerbation. The present study describes, for the first time, the detection of rhinovirus from induced sputum that samples lower airway secretions in patients with COPD exacerbation and investigates the relationship of rhinovirus infection to symptoms and lower airway cytokine production during exacerbation.

\section{Method}

\section{Study design}

This was a prospective study of COPD patients, in which samples were taken from patients at clinic during at least one exacerbation and in the stable state (baseline samples). Patients were monitored daily by diary cards, in which they recorded any increase in their chronic (i.e. stable) symptoms, "major" (dyspnoea, sputum purulence 
and sputum amount) and "minor" (increased nasal discharge/increased nasal congestion, wheeze, sore throat and cough), during the last $24 \mathrm{~h}[3-5,10]$. Diary cards were inspected at each clinic for evidence of exacerbation as defined below. Patients were considered to have rhinovirus infection if either the induced sputum or the nasal aspirate was positive for rhinovirus at COPD exacerbation.

\section{Recruitment}

Thirty three (25 male) patients with COPD were recruited from the outpatients department of the London Chest Hospital. Inclusion criteria were: forced expiratory volume in one second (FEV1) of $<70 \%$ of the predicted value for age and height, a history of $\beta_{2}$-agonist reversibility of $<15 \%$ and/or $200 \mathrm{~mL}$ with respect to baseline FEV1 [11], and an absence of a history of asthma, bronchiectasis, carcinoma of the bronchus or other significant respiratory disease. Patients were immunized against the influenza virus. Ethical permission was obtained from the East London and City Health Authority Ethics Committee and informed consent was obtained from each patient.

\section{Baseline visit}

Patients were seen during a symptom-free period $>3$ weeks after the last COPD exacerbation. At this visit, measurements were made of height, FEV1 and forced vital capacity (FVC) by rolling seal spirometer (Sensor Medic Corporation, Yorba Linda, CA, USA), and FEV1 reversibility to $400 \mu \mathrm{g}$ inhaled salbutamol administered via metered-dose inhaler and spacer. Arterialized ear lobe blood gas analysis was performed [12] and nasopharyngeal and induced sputum samples were taken as described below. Stable daily symptoms, the number of exacerbations in the previous 12 months and drug history were noted. The number of cigarettes smoked daily and the duration of smoking (in years) were recorded and the cumulative cigarette exposure (in pack-yrs; cigarettes. day $^{-1} \times$ yrs/20) calculated [20].

\section{Exacerbations}

Exacerbations were diagnosed according to defined criteria, modified from ANTHONISEN et al. [10] and validated and used in previous studies [3-5]. Exacerbations were identified from symptoms recorded on the diary cards by the appearance or deterioration of any two of the aforementioned major symptoms or one major and one minor symptom occurring on two consecutive days, the first of which was called the day of onset of exacerbation [5]. When patients experienced deterioration in their daily symptoms, they were required to call a member of the clinical team (T.A.R. Seemungal or A. Bhowmilk) and were seen at the outpatients department within $48 \mathrm{~h}$, before any treatment for the exacerbation was administered. If an exacerbation of COPD was diagnosed in the patient then investigations were performed as outlined below. Symptoms on the day of presentation were binary coded and summed to give a symptom count.

\section{Clinical specimens}

Nasopharyngeal samples. At each visit, baseline or exacerbation, nasopharyngeal samples were taken using a sterile mucus trap attached to a $\mathrm{CH} 10$ nasal catheter (Maerst Medical, Hundested, Denmark) which was placed high in the anterior nares. The mucus trap was connected to a portable suction apparatus. If the volume of nasal aspirate was $<0.5 \mathrm{~mL}$, rhinorrhoea was induced by spraying $0.1 \mathrm{~mL}$ $8 \mathrm{mg} \cdot \mathrm{mL}^{-1}$ histamine (BDH Chemicals, Poole, UK) in $0.9 \%$ sodium chloride (made up freshly each day) into each nostril [6]. If the volume of nasal aspirate was still low, swabs of the anterior nares, pharynx and tonsils were taken and immediately immersed in phosphate-buffered saline. Both nasal aspirates and swabs were stored at $4{ }^{\circ} \mathrm{C}$ and transported to the laboratory within $4 \mathrm{~h}$. At the laboratory, phosphate-buffered saline $(0.172 \mathrm{M} ; \mathrm{pH}$ : 7.3) was added to each aspirate or swab to a final volume of $3.25 \mathrm{~mL}$ and $0.25-\mathrm{mL}$ aliquots were stored at $-70^{\circ} \mathrm{C}$ for further analysis by PCR. One aliquot was taken for rhinovirus culture.

Induced sputum. Induced sputum at exacerbation and baseline was obtained using standard methods validated in the authors' laboratory $[4,13]$. Briefly, after premedication with $200 \mu \mathrm{g}$ inhaled salbutamol via a metered-dose inhaler with spacer, nebulization with $3 \%$ saline was commenced using the DeVilbiss UltraNeb 2000 ultrasonic nebulizer (DeVilbiss Healthcare, Heston, UK). Nebulization was continued for $14 \mathrm{~min}$ and patients were instructed to blow their noses and rinse their mouths out with water before expectorating sputum into a sterile pot.

\section{Laboratory analysis}

Cell counts. Induced sputum samples were examined as soon as possible within $2 \mathrm{~h}$ after sampling, adopting methods previously evaluated $[4,13]$. The selected portion of sputum was placed in a preweighed tube and the weight of the selected portion of sputum recorded. One portion of the selected sputum was homogenized with 4 times its weight of $0.1 \%$ dithiothreitol (DTT) and then Hank's balanced salt solution was added to produce a final 10 -fold dilution of sputum. This was then centrifuged for $10 \mathrm{~min}(700 \times g)$ at 2,000 revolutions per minute (rpm) to yield a cell pellet for total and differential cell counts and the supernatant was used for cytokine assay. The cell pellet was used to prepare cytospin slides that were then stained with Diff-Quik (Dade-Behring, Dödingen, Switzerland) in order to obtain differential cell counts, performed by counting 400 cells per slide. The other portion of the selected sputum was homogenized with glass beads and phosphate-buffered saline to produce a 10 -fold dilution that was used for the viral PCR.

Cytokine estimation. Samples were analysed at the end of the study in a manner blinded as to the presence or absence of exacerbation. Interleukin (IL)-6 and IL-8 in the supernatant of the induced sputum samples were measured using a quantitative sandwich enzyme-linked immunosorbent assay (R\&D Systems Europe, Abingdon, UK). A monoclonal antibody specific to each cytokine was precoated on microtitre plates and standards and samples added. After washing, an enzyme-linked polyclonal antibody was added, with a substrate solution for colour development and intensity reading.

Rhinovirus ribonucleic acid extraction and reverse transcription. Nasopharyngeal and induced sputum samples 
were analysed using a reverse transcriptase (RT)-PCR method with rhinovirus-specific primers $[14,15]$. In a class I microbiological safety cabinet, $250 \mu \mathrm{L}$ nasal aspirate or induced sputum were added to $200 \mu \mathrm{L}$ Trizol $\mathbb{R}$ Reagent (Life Technologies, Paisley, UK), the mixture agitated on a vortex mixer for $30 \mathrm{~s}$ and then placed on ice. Chloroform (50 $\mu \mathrm{L}$; Sigma Chemicals, Poole, UK) was then added, the mixture agitated on a vortex mixer for $30 \mathrm{~s}$ and allowed to stand on ice for $10 \mathrm{~min}$. Samples were then centrifuged $(12,000 \times g)$ for $5 \mathrm{~min}$; the aqueous phase was removed and placed in a clean tube containing $2 \mu \mathrm{L} 20 \mathrm{mg} \cdot \mathrm{mL}^{-1}$ glycogen (type VII; Sigma Chemicals) in water. The volume was adjusted to $1.5 \mathrm{~mL}$ using isopropanol (Sigma Chemicals) and the mixture agitated on a vortex mixer for $15 \mathrm{~s}$. Samples were left at $-70^{\circ} \mathrm{C}$ for $18 \mathrm{~h}$ and then centrifuged $(14,000 \times g)$ for $25 \mathrm{~min}$. The supernatant was then discarded, and $80 \mu \mathrm{L} 80 \%$ (volume/ volume) ethanol in water added to the RNA pellet. Samples were centrifuged $(12,000 \times g)$ for $5 \mathrm{~min}$, the supernatant was discarded and the resulting RNA pellet gently heat-dried for 5 min or until liquid was no longer visible. RNA pellets were resuspended in a warmed $\left(37^{\circ} \mathrm{C}\right.$ ) mixture of $10 \mu \mathrm{L}$ water (molecular biology grade; Sigma Chemicals) and $1 \mu \mathrm{L} 65 \mathrm{U}$ RNAguard R (Amersham Pharmacia Biotech, St Albans, UK). A mixture of $8 \mu \mathrm{L}$ water (molecular biology grade) and $2 \mu \mathrm{L}(500$ $\mu \mathrm{g} \cdot \mathrm{mL}^{-1}$ ) random hexamers (Promega UK Ltd., Southampton, UK) was then added to the samples and heated to $70^{\circ} \mathrm{C}$ for $10 \mathrm{~min}$. Samples were quenched on ice, and then the following mixture was added: $8 \mu \mathrm{L} 5 \times$ First Strand Buffer (Life Technologies), $4 \mu \mathrm{L}(0.02 \mathrm{M}) \mathrm{DTT}$ (Life Technologies), $1 \mu \mathrm{L}(1 \mathrm{mM})$ deoxyribonucleoside triphosphates (dNTPs) (Promega UK Ltd.), $5 \mu \mathrm{L}$ water (molecular biology grade) and $2 \mu \mathrm{L} 200 \mathrm{U}$ SUPERSCRIPT Reverse Transcriptase (Life Technologies). Reverse transcription was performed for $1 \mathrm{~h}$ at $37^{\circ} \mathrm{C}$. The resulting complementary deoxyribonucleic acid (DNA) samples were stored at $4{ }^{\circ} \mathrm{C}$ if PCR was performed on the same day, or at $-20^{\circ} \mathrm{C}$ if PCR was performed at any other time.

Polymerase chain reaction. PCR was performed in a $50-\mu \mathrm{L}$ reaction volume consisting of $5.6 \mu \mathrm{L} 10 \times$ Reaction Buffer (Advanced Biotechnologies Formula, Advanced Biotechnologies, Epsom, UK), $5.6 \mu \mathrm{L}(2.8 \mathrm{mM}) \mathrm{MgCl}_{2}$ (Advanced Biotechnologies), $0.5 \mu \mathrm{L}(0.2 \mathrm{mM}) \mathrm{dNTPs}$, $0.5 \mu \mathrm{L}(1.42 \mu \mathrm{M})$ oligonucleotide primer OL26, $0.5 \mu \mathrm{L}$ $(1.59 \mu \mathrm{M})$ OL27 and $0.25 \mu \mathrm{L}(1.25 \mathrm{U}) \mathrm{Taq}$ DNA Polymerase (Advanced Biotechnologies) adjusted to $45 \mu \mathrm{L}$ with water (molecular biology grade) [13]. cDNA $(5 \mu \mathrm{L})$ was added to the reaction mixture and PCR performed as follows: 1 cycle at $94^{\circ} \mathrm{C}$ for $5 \mathrm{~min}, 53^{\circ} \mathrm{C}$ for $30 \mathrm{~s}$ and $72^{\circ} \mathrm{C}$ for $1 \mathrm{~min}, 40$ cycles at $94^{\circ} \mathrm{C}$ for $1 \mathrm{~min}, 53^{\circ} \mathrm{C}$ for $30 \mathrm{~s}$ and $72^{\circ} \mathrm{C}$ for $1 \mathrm{~min}$, and then final extension at $72^{\circ} \mathrm{C}$ for $5 \mathrm{~min}$. Rhinovirus was identified by the presence of a 380-base pair product on ethidium bromide-stained $2 \%$ (weight/ volume) agarose gel electrophoresis. PCR was repeated at least three times to confirm the result and a sample was taken as positive only if the PCR result was positive on all three occasions.

Deoxyribonucleic acid sequencing. Positive PCR was confirmed by sequencing of the product. Template DNA was amplified in a 40-cycle PCR using the primers OL26 and OL27 in a total reaction volume of $100 \mu \mathrm{L}$ as described above. These products were purified using the QIAquick PCR purification kit (Qiagen, Crawley, UK) and a cycle sequencing reaction using the primer OL26 and the ABI PRISM $^{\mathrm{TM}}$ Dye Terminator Ready Reaction Kit (Perkin Elmer, Warrington, UK). Products were sequenced on an ABI 377 Automated Sequencer and the resulting sequences compared to those in the GenBank database [16].

Rhinovirus culture. Nasopharyngeal samples were cultured in human diploid fibroblasts at 33 and $37^{\circ} \mathrm{C}$ in duplicate tubes. Cultures were examined every other day for cytopathic effects, which were confirmed by acid stability testing for rhinovirus. Cultures were observed for 14 days.

\section{Statistical analysis}

Normally distributed data are presented as mean \pm SD, skewed data as median (interquartile range (IQR)), and categorical data as percentage and odds ratio. Comparisons between subgroups of normally distributed continuous variables were analysed using analysis of variance. Continuous variables with normal distributions were compared using a t-test, whereas those with non-normal distributions were compared using the Mann-Whitney U-test or Wilcoxon signed-rank test as appropriate and categorical variables using the Chi-squared test. Spearman's rank correlation was used to study relationships of variables to induced sputum IL-6 levels. Data were available for 43 exacerbations in 33 patients. The same baseline was used for repeated exacerbations in the same patient. Differences between baseline and exacerbation were examined using a paired t-test or Wilcoxon signed-rank test and differences between rhinovirus and nonrhinovirus-associated exacerbations by means of an independent samples unpaired ttest or Mann Whitney U-test as appropriate. Statistical analysis was carried out using the computer software SPSS 7.5 for windows (SPSS Inc., Chicago, IL, USA).

\section{Results}

\section{Patients}

Patients had moderate-to-severe COPD and were sampled during 43 exacerbations. These patients were all smokers, with 13 (39.4\%) being current smokers (table 1). They all received a mean \pm SD inhaled steroids dose of $1.26 \pm 0.74 \mathrm{~g} \cdot \mathrm{day}^{-1}$. The patients experienced three (two to four) (median (IQR)) exacerbations in the year prior to recruitment. Induced sputum samples at baseline were obtained from 22 of the 33 patients and these 22 patients did not differ from the 11 patients who did not provide baseline induced sputum samples in any of the characteristics mentioned in table 1 ( $p>0.05$ in all cases).

\section{Detection of rhinovirus at exacerbation and baseline}

From the 43 exacerbations, 42 paired induced sputum and nasopharyngeal specimens were obtained. For the remaining exacerbation, the induced sputum sample for PCR analysis was misplaced during storage but a nasopharyngeal sample was obtained. A total of 10 (23\%) exacerbations were associated with rhinovirus in either induced sputum or nasopharyngeal samples. Rhinovirus was detectable in the induced sputum of all of these but 
Table 1. - Characteristics of the 33 patients in the study

\begin{tabular}{lcc}
\hline & Mean & SD \\
\hline Age yrs & 65.4 & 8.20 \\
FEV1 L & 1.07 & 0.36 \\
FVC L & 2.58 & 0.71 \\
FEV $1 / \mathrm{FVC}$ & 0.44 & 0.17 \\
FEV1 \% pred* & 40.0 & 16.0 \\
Reversibility \% prebronchodilator FEV1 & 10.8 & 10.6 \\
$\mathrm{~Pa}_{2} \mathrm{O}_{2} \mathrm{kPa}$ & 9.06 & 0.92 \\
$\mathrm{~Pa}, \mathrm{CO}_{2} \mathrm{kPa}$ & 5.90 & 0.70 \\
Cumulative cigarette exposure pack-yrs & 45.9 & 34.7 \\
Smoking duration yrs & 39.4 & 13.8 \\
Time on inhaled steroids yrs & 3.67 & 2.52 \\
\hline
\end{tabular}

*: for age and height. FEV1: forced expiratory volume in one second; FVC: forced vital capacity; $\mathrm{Pa}_{\mathrm{a}} \mathrm{O}_{2}$ : arterial oxygen tension; $P \mathrm{a}_{1} \mathrm{CO}_{2}$ : arterial carbon dioxide tension; \% pred: percentage of the predicted value.

one, for which no sample was available. In four $(40 \%)$ of these, rhinovirus was not detected in the nasopharyngeal sample, despite being detected in the induced sputum by PCR. Of the 10 samples positive for rhinovirus by PCR, only one was isolated by viral culture.

The baseline nasopharyngeal samples obtained from all 33 patients and the 22 baseline induced sputum samples were all negative for rhinovirus by PCR and rhinovirus culture.

\section{Symptoms, spirometry and rhinovirus detection}

Increased nasal discharge/nasal congestion ("colds") was present in $26(61 \%)$ of the exacerbations and sore throat in $13(30 \%)$. Thirty seven $(86 \%)$ exacerbations were associated with increased dyspnoea, $11(25 \%)$ with increased sputum purulence, $29(67 \%)$ with increased sputum volume, 21 (49\%) with increased wheeze and 20 (47\%) with increased cough. The simultaneous presence of a cold and increased sputum volume at exacerbation was the symptom combination that was most strongly associated with the presence of rhinovirus (table 2). However, two $(20 \%)$ exacerbations positive for rhinovirus were not associated with nasal symptoms and these patients complained of increased dyspnoea, increased wheeze, increased cough and no sputum changes. At onset of COPD exacerbation, the median (IQR) total symptom score for rhinovirus-associated exacerbations was 4 (3-6), which was greater than the median total symptom score of 3 (34) for nonrhinovirus-associated exacerbations $(p=0.039)$.

The mean \pm SD FEV 1 decreased from $1.084 \pm 0.320 \mathrm{~L}$ at baseline to $0.989 \pm 0.299 \mathrm{~L}$ at exacerbation, and the FVC decreased from $2.916 \pm 0.972$ to $2.601 \pm 0.736 \mathrm{~L}$ ( $p<0.001$ in both cases). However there was no relationship between FEV1 or FVC change at exacerbation and the presence or absence of rhinovirus.

\section{Induced sputum and rhinoviruses}

The median (IQR) IL-6 concentration increased from $90.2(51.6-149.1) \mathrm{pg} \cdot \mathrm{mL}^{-1}$ at baseline to $140.3(49.8-$ $318.0) \mathrm{pg} \cdot \mathrm{mL}^{-1}$ at exacerbation $(\mathrm{p}=0.005)$. However, the change in IL-8 concentration from $6,448(2,795-11,921)$ $\mathrm{pg} \cdot \mathrm{mL}^{-1}$ at baseline to $5,540(1,828-13,033) \mathrm{pg} \cdot \mathrm{mL}^{-1}$ at exacerbation was not significant $(\mathrm{p}=0.60)$. Total and differ-
Table 2. - Association of changes in respiratory symptoms with detection of rhinovirus for 43 exacerbations in 33 chronic obstructive pulmonary disease patients

\begin{tabular}{lcc}
\hline Symptom & Odds ratio* & p-value \\
\hline Increased dyspnoea & 0.24 & 0.112 \\
Increased sputum purulence & 1.33 & 0.715 \\
Increased sputum volume & 5.84 & 0.112 \\
Colds $^{+}$ & 3.33 & 0.164 \\
Increased wheeze & 1.06 & 0.933 \\
Sore throat & 5.57 & 0.026 \\
Cough & 2.03 & 0.334 \\
Colds $^{+}$with increased sputum volume & 6.15 & 0.036 \\
\hline
\end{tabular}

*: determined from univariate logistic regression with presence of rhinovirus as outcome variable; ${ }^{+}$: increased nasal discharge/ nasal congestion.

ential macrophage, eosinophil and neutrophil counts did not change significantly between baseline and exacerbation. However, the baseline total lymphocyte count. Increased from $0.044(0.028-0.104) \times 10^{6}$ cells. $g$ sputum $^{-1}$ to $0.100(0.030-0.165) \times 10^{6}$ cells $\cdot g$ sputum $^{-1}$ at exacerbation $(\mathrm{p}=0.040)$.

Table 3 shows that there was a tendency for the sputum IL-6 concentration at baseline in the nonrhinovirusinfected group to be lower than that in the rhinovirusinfected group. The IL- 6 concentration was twice as high during rhinovirus-associated exacerbations as compared to nonrhinovirus-associated exacerbations, although this difference was not statistically significant $(p=0.25)$ However, as shown in figure 1, table 3 , the change in IL-6 concentration at exacerbation compared to baseline was significantly higher during rhinovirus infection than in its absence $(p=0.008)$. IL- 8 concentration and total cell counts did not differ significantly between rhinovirusassociated and nonrhinovirus-associated exacerbations ( $p>0.40$, in all cases).

\section{Discussion}

In the present study, it was found that rhinovirus was associated with $23 \%$ of COPD exacerbations; the virus being detected with higher frequency in induced sputum from the lower airway as compared to upper airway specimens. Rhinovirus was associated with a greater rise in lower airway IL-6 levels and total symptom score at COPD exacerbation. This is the first study to detect such a high incidence of rhinovirus infection during COPD exacerbations and also the first to show that wild-type rhinovirus is very likely to infect the lower airway in COPD patients. Positive PCR reactions were confirmed by DNA sequencing, although previous studies of natural rhinovirus infection have not used sequencing $[6,17,18]$.

Viruses are important triggers of COPD exacerbation $[9$, 19] but rhinovirus has not hitherto been considered to be of much significance during exacerbations of COPD. In a study of 44 chronic bronchitics over 2 yrs, STOTт et al. [19] found rhinovirus in $13(14.9 \%)$ of 87 exacerbations of chronic bronchitis using viral culture methods. In a more detailed study of 25 chronic bronchitics with 116 exacerbations over 4 yrs and using similar methods, GuMP et al. [9] found that only $3.4 \%$ of exacerbations could be attributed to rhinovirus. In a more recent study of 35 
Table 3. - Differences in symptoms and induced sputum cytokines between rhinovirus and nonrhinovirus-associated chronic obstructive pulmonary disease exacerbations

\begin{tabular}{|c|c|c|c|c|}
\hline & Exacerbations $\mathrm{n}$ & No rhinovirus & Rhinovirus & p-value* \\
\hline \multicolumn{5}{|l|}{ IL-6 } \\
\hline At baseline & 22 & $120.3(12.7-207.5)$ & $90.2(75.9-95.2)$ & 0.733 \\
\hline At exacerbation & 43 & $128.5(54.3-157.3)$ & $249.6(100.0-349.5)$ & 0.250 \\
\hline Change at exacerbation & 30 & $7.2(-19.7-75.1)$ & $248.0(44.1-275.2)$ & 0.008 \\
\hline \multicolumn{5}{|l|}{ IL-8 } \\
\hline At baseline & 22 & $6447(729-12331)$ & 6925 (2369-11236) & 0.865 \\
\hline At exacerbation & 43 & $6178(1699-13091)$ & 3732 (1901-17265) & 0.625 \\
\hline Change at exacerbation & 30 & $68(-3637-5952)$ & $1964(-2987-3506)$ & 0.924 \\
\hline
\end{tabular}

Data are presented as median (interquartile range). *: Mann-Whitney U-test. IL: interleukin.

episodes of COPD exacerbation using serological methods and nasal samples for viral culture, little evidence was found for a rhinovirus aetiology of COPD exacerbation [20]. Other viruses may trigger COPD exacerbation, although coronavirus was associated with only a small proportion of asthmatic exacerbations $[6,18]$ and all the COPD patients in the present study had undergone influenza immunization. Although bacteria such as Haemophilus influenzae and Streptococcus Pneumoniae have been associated with COPD exacerbation, recent studies have not shown increased bacterial counts during exacerbation [21].

Conventional diagnostic methods for rhinovirus infection, discussed above, were limited by the poor sensitivity of cell cultures for rhinovirus. Serological diagnosis of rhinovirus is hampered by the large number of serotypes $(>100)$ and limited cross-reactivity [22]. Recently, it has been found that detection of rhinovirus could be improved by up to five times using PCR [14, 23]. Thus, using PCR, rhinovirus has been shown to be the predominant virus, associated with $50 \%$ of asthmatic exacerbations in children and $33 \%$ in adults and $19 \%$ of exacerbations of cystic fibrosis, with smaller proportions of exacerbations attributed to other viruses [6, 18, 24]. Using limiting dilutions of human rhinovirus 14, the PCR

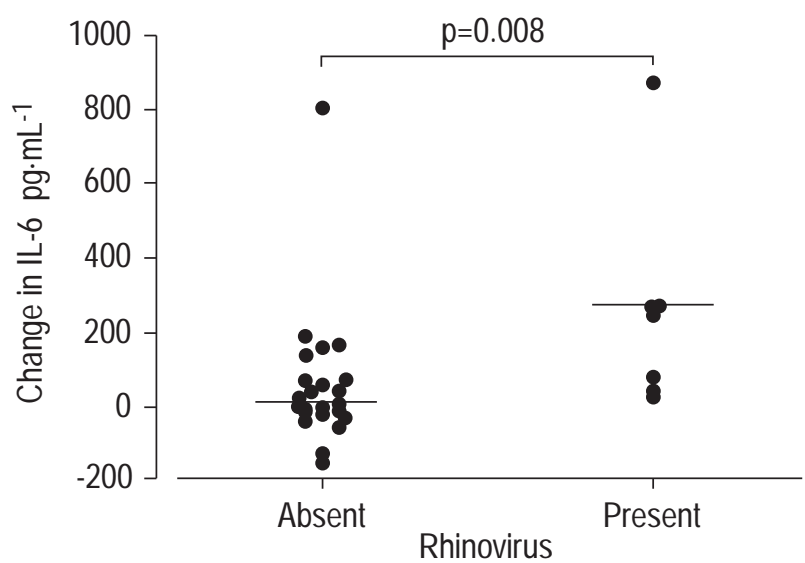

Fig. 1. - Change in induced sputum interleukin-6 (IL-6) concentration from baseline to exacerbation in 30 exacerbations in 22 chronic obstructive pulmonary disease patients with seven exacerbations due to rhinovirus compared to 23 nonrhinovirus exacerbations. Medians are shown as horizontal bars; comparison is by Mann-Whitney U-test. (If both outliers are excluded the difference between the two groups is still significant $(\mathrm{p}=0.01))$. used in the present study has been shown to have a detection limit of $1 \times 10^{3}$ median tissue culture infective doses $\cdot \mathrm{mL}^{-1}$ by gel electrophoresis [14]. In the present study, only one of the 10 samples rhinovirus-positive by PCR yielded positive results by viral culture methods, again demonstrating the superiority of PCR techniques. However, all previous studies of naturally acquired rhinovirus infection have used upper airway samples (either throat swabs or nasal aspirates) and no previous comparison has been made between upper and lower airway samples using induced sputum as in the present study. Rhinovirus was detected in four induced sputum samples in the present study but not in the corresponding nasopharyngeal samples, giving a $40 \%$ higher detection rate for rhinovirus using induced sputum and suggesting that rhinovirus may directly infect the lower airway. These results also indicate that induced sputum is suitable and preferable for rhinovirus detection and are consistent with those of Horn et al. [25] who showed that viruses were more frequently detected in sputum than nasal and throat swabs in children with wheezy bronchitis. Contamination of lower airway specimens by upper airway secretions cannot entirely be ruled out, but, as induced sputum samples the lower airway, the present study contributes to evidence that naturally acquired rhinovirus infection may infect the lower airway.

COPD exacerbation is defined by clinical criteria [3-5, $10]$ although these are not considered useful for determining the cause of the exacerbation [26]. An exacerbation was regarded as having occurred when there were at least two symptoms, at least one of which was major, thus the presence of a cold as an upper airway symptom by itself did not constitute an exacerbation [3-5]. However, in the current study, it was shown that the symptom combination most strongly associated with rhinovirus detection was a cold with increased sputum, and rhinovirus infection was associated with a greater total symptom score at COPD exacerbation. Furthermore, $20 \%$ of the patients with rhinovirus infection had no nasal symptoms. No differences in spirometric changes were found between rhinovirus and nonrhinovirus COPD exacerbation, although common colds have been associated with changes in lower airway resistance in normal patients [27]. Changes in lower airway inflammatory indices have been associated with experimental rhinovirus infection [7, 28]. It has previously been shown that there are increased inflammatory changes in the lower airway during COPD exacerbation [4]. As in the previous study, it was found 
that induced sputum IL-6 levels increased significantly at exacerbation compared to baseline, although there were no corresponding changes in IL-8 concentration or cell counts [4]. Rhinovirus detection was associated with the presence of increased IL-6 concentration at exacerbation compared to baseline but not with IL-8 changes, which were variable. Lower airway IL-8 levels have been shown to increase with experimental rhinovirus type 16 infection in normal and asthmatic patients in some studies [29], but not in others [28]. However, COPD patients already have upregulated airway IL-8 levels when stable, possibly due to their high sputum neutrophil load [30], and so a further increase in IL-8 concentration at COPD exacerbation would be unlikely.

Using a transformed epithelial cell line, rhinovirus has been shown to attach to airway epithelium [31] and to increase IL-6 production from epithelial cells through a nuclear factor- $\kappa \mathrm{B}$-dependent transcription pathway [32]. This could explain the increased IL- 6 levels in induced sputum from patients with rhinovirus infection in the present study. Experimental rhinovirus infection has been shown to cause increased IL-6 levels in nasal washings, which were related to symptom scores [33]. In the present study, it was found that total symptom score was related to rhinovirus detection but not to IL-6 levels. Experimental rhinovirus infection has also been shown to cause increased inflammatory marker levels in normal subjects and asthmatics, with increases in sputum IL-6 concentration $[7,28,29]$, although COPD patients have not been studied as it is not possible to infect patients with COPD in view of their restricted lung function. Thus IL-6 may play an important part in the pathogenesis of rhinovirus infection. IL-6 also plays a central role in the host response to stress and injury through its ability to induce the acute phase response. However, IL-6 may also have effects on B-cell differentiation, stimulate mucosal immunoglobulin A responses and function as a costimulator of T-cells. Thus increased IL-6 levels may protect against further human rhinovirus infection and this may explain the tendency of patients in the present study with lower IL-6 levels at baseline to become infected with rhinovirus. Susceptibility to rhinovirus infection may be increased by the cytokine tumour necrosis factor- $\alpha$ and reduced by interferon gamma $[32,34]$, although the effect of IL-6 has not been previously evaluated.

Human rhinovirus infection was associated with increased induced sputum interleukin-6 levels, which may play a role in producing lower airway inflammation and symptom changes associated with chronic obstructive pulmonary disease exacerbation. Interleukin-6 may have an important role in the pathogenesis of rhinovirus infection of the lower airway in chronic obstructive pulmonary disease, although further studies are required to evaluate the mechanisms involved. Treatment aimed at preventing rhinovirus-induced airway inflammation would have a significant impact on morbidity and mortality in chronic obstructive pulmonary disease patients.

Acknowledgements. The authors would like to thank S. Johnston, Imperial College at St. Mary's Hospital, London, for helpful advice on polymerase chain reaction techniques for rhinovirus detection.

\section{References}

1. Fletcher C, Peto R. The natural history of chronic airflow obstruction. BMJ 1977; 1: 1645-1648.

2. Kanner RE, Renzetti AD Jr, Klauber MR, Smith CB, Golden CA. Variables associated with changes in spirometry in patients with obstructive lung diseases. Am J Med 1979; 67: 44-50.

3. Seemungal TAR, Donaldson DC, Paul EA, Bestall JC, Jeffries DJ, Wedzicha JA. Effect of exacerbation on quality of life in patients with chronic obstructive pulmonary disease. Am J Respir Crit Care Med 1998; 157 : $1418-1422$.

4. Bhowmik A, Seemungal TAR, Sapsford RJ, Wedzicha JA. Relation of sputum inflammatory markers to symptoms and physiological changes at COPD exacerbation. Thorax 2000; 55: 114-120.

5. Seemungal TAR, Donaldson GC, Bhowmik A, Jeffries DJ, Wedzicha JA. Time course and recovery of exacerbations in patients with chronic obstructive pulmonary disease. Am J Respir Crit Care Med 2000; 16: 16081618.

6. Johnston SL, Pattemore PK, Sanderson G, et al. Community study of the role of viral infections in exacerbations of asthma in 9-11 year old children. BMJ 1995; 310: 1225-1229.

7. Fraenkel DJ, Bardin PG, Sanderson G, et al. Lower airways inflammation during rhinovirus colds in normal and in asthmatic subjects. Am J Respir Crit Care Med 1995; 151: 879-886.

8. Gern JE, Galagan DM, Jarjour NN, Dick EC, Busse W. Detection of rhinovirus RNA in lower airway cells during experimentally induced infection. Am J Respir Crit Care Med 1997; 155: 1159-1161.

9. Gump DW, Phillips CA, Forsyth BR, McIntosh FK, Lamborn KR, Stouch WH. Role of infection in chronic bronchitis. Am Rev Respir Dis 1976; 113: 465-473.

10. Anthonisen NR, Manfreda J, Warren CPW, Hershfield ES, Harding GKM, Nelson NA. Antibiotic therapy in exacerbations of chronic obstructive pulmonary disease. Ann Intern Med 1987; 106: 196-204.

11. American Thoracic Society Statement. Standards for the diagnosis and care of patients with chronic obstructive pulmonary disease. Am J Respir Crit Care Med 1995; 152: S78-S83.

12. Pitkin AD, Roberts CM, Wedzicha JA. Arterialised ear lobe blood gas analysis: an underused technique. Thorax 1994; 49: 364-366.

13. Bhowmik A, Seemungal TAR, Sapsford RJ, Devalia JL, Wedzicha JA. Comparison of spontaneous and induced sputum for investigation of airway inflammation in chronic obstructive pulmonary disease. Thorax 1998; 53: 953-956.

14. Johnston SL, Sanderson G, Pattemore PK, et al. Use of polymerase chain reaction for diagnosis of picornavirus infection in subjects with and without respiratory symptoms. J Clin Micro 1993; 31: 111-117.

15. Gama RE, Horsnell PR, Hughes PJ, et al. Amplification of rhinovirus specific nucleic acids from clinical samples using the polymerase chain reaction. J Med Virol 1989; 28: 73-77.

16. Benson DA, Boguski MS, Lipman DJ, et al. GenBank. Nucleic Acids Research 1999; 27: 12-17.

17. Pizzichini MMM, Pizzichini E, Efthimiadis A, et al. Asthma and natural colds. Am J Respir Crit Care Med 1998; 158: 1178-1184.

18. Nicholson KG, Kent J, Ireland DC. Respiratory viruses 
and exacerbations of asthma in adults. BMJ 1993; 307 : 982-986.

19. Stott EJ, Grist NR, Eadie MB. Rhinovirus infections in chronic bronchitis: isolation of eight possible new rhinovirus serotypes. J Med Microbiol 1968; 1: 109117.

20. Philit F, Etienne J, Calvet A, et al. Infectious agents associated with exacerbations of chronic obstructive pulmonary disease and attacks of asthma. Rev Mal Respir 1992; 9: 191-196.

21. Wilson R. Bacterial infection and chronic obstructive pulmonary disease. Eur Respir J 1999; 13: 233-235.

22. Hamparian VV, Colonno RJ, Cooney MK, et al. A collaborative report: rhinoviruses - extension of the numbering system from 89-100. Virology 1987; 159: 191-192.

23. Ireland DC, Kent J, Nicholson KG. Improved detection of rhinoviruses in nasal and throat swabs by semi-nested RTPCR. J Med Virol 1993; 40: 96-101.

24. Collinson J, Nicholson KG, Cancio E, et al. Effects of upper respiratory tract infections in patients with cystic fibrosis. Thorax 1996; 51: 115-122.

25. Horn ME, Reed SE, Taylor P. Role of viruses and bacteria in acute wheezy bronchitis in childhood: a study of sputum. Arch Dis Child 1979; 54: 587-592.

26. Wijnands GJ. Diagnosis and interventions in lower respiratory tract infections. Am J Med 1992; 82: 91S-97S.

27. Picken JJ, Niewoehner DE, Chester EH. Prolonged effects of viral infections of the upper respiratory tract upon small airways. Am J Med 1971; 52: 738-746.

28. Fleming HE, Little EF, Schnurr D, et al. Rhinovirus-16 colds in healthy and in asthmatic subjects. Am J Respir Crit Care Med 1999; 160: 100-108.

29. Grunberg K, Smits HH, Timmers MC, et al. Experimental rhinovirus 16 infection: effects on cell differentials and soluble markers in sputum in asthmatic subjects. $A m J$ Respir Crit Care Med 1997; 156: 609-616.

30. Keatings VM, Collins PD, Scott DM, Barnes PJ. Differences in interleukin-8 and tumour necrosis factor-alpha in induced sputum from patients with chronic obstructive pulmonary disease or asthma. Am J Respir Crit Care Med 1996; 153: 530-534.

31. Johnston SL, Papi A, Bates PJ, Mastronarde JG, Monick MM, Hunninghake GW. Low grade rhinovirus infection induces a prolonged release of IL-8 in pulmonary epithelium. J Immunol 1998; 160: 6172-6181.

32. Subauste MC, Jacoby DB, Richards SM, Proud D. Infection of a human respiratory epithelial cell line with rhinovirus. J Clin Invest 1995; 96: 549-557.

33. Zhu Z, Tang W, Ray A. Rhinovirus stimulation of Interleukin-6 in vivo and in vitro. J Clin Invest 1996; 97: 421430.

34. Gern JE, Vritis R, Kelly EAB, Dick EC, Busse WW. Rhinovirus produces nonspecific activation of lymphocytes through a monocyte-dependent activation. $J$ Immunol 1996; 157: 1605-1612. 